\title{
Distortion-Free Magnetic Resonance Imaging in the Zero-Field Limit
}

Nathan Kelso ${ }^{1,3}$, Seung-Kyun Lee ${ }^{1,3^{*}}$, Louis-S. Bouchard ${ }^{2,3 \dagger}$, Vasiliki Demas ${ }^{2,3 \ddagger}$, Michael Mück $^{4}$, Alexander Pines ${ }^{2,3}$, and John Clarke ${ }^{1,3 \S}$

Departments of Physics ${ }^{1}$ and Chemistry ${ }^{2}$, University of California, Berkeley, CA 94720 Materials Sciences Division, Lawrence Berkeley National Laboratory ${ }^{3}$, Berkeley, CA 94720

Institut für Angewandte Physik, Justus-Leibig-Universität Gießen ${ }^{4}$, D-35392 Gießen, Germany

* Current address: GE Global Research, One Research Circle, Niskayuna, NY 12309

$\dagger$ Current address: Department of Chemistry and Biochemistry, 607 Charles E. Young

Dr. East, University of California, Los Angeles, CA 90095

† Current address: T2 Biosystems, 286 Cardinal Medeiros Ave, Cambridge, MA 02141

$\S$ To whom correspondence should be addressed. Email: jclarke@berkeley.edu 
Magnetic resonance imaging ${ }^{1,2}(\mathrm{MRI})$ is a powerful technique for clinical diagnosis and materials characterization. Images are acquired in a homogeneous static magnetic field much higher than the fields generated across the field of view by the spatially encoding field gradients ${ }^{3,4}$. Without such a high field, the concomitant components of the field gradient dictated by Maxwell's equations lead to severe, essentially intractable distortions ${ }^{5-7}$ that make imaging impossible with conventional MRI encoding. In this paper, we present a distortion-free image of a phantom acquired with a fundamentally different methodology ${ }^{8}$ in which the applied static field approaches zero. Our technique involves encoding with pulses of uniform and gradient field, and acquiring the magnetic field signals with a SQUID ${ }^{9}$. The method can be extended to weak ambient fields, potentially enabling imaging in the Earth's field without cancellation coils or shielding. Other potential applications include quantum information processing ${ }^{10,11}$ and fundamental studies of long-range ferromagnetic interactions ${ }^{12}$.

In MRI, the Larmor precession frequency $\omega(x, y, z)=\gamma B(x, y, z)$ of the proton spins in the position-dependent magnetic field $B(x, y, z)$ frequency- and phase-encodes the proton density distribution into a magnetic signal that is subsequently decoded to form an image ${ }^{4}\left(\gamma\right.$ is the magnetogyric ratio). In clinical MRI machines ${ }^{4}$ the strength of the applied homogeneous static magnetic field $\boldsymbol{B}_{0}=B_{0} \hat{\mathbf{z}}$ is typically $1.5 \mathrm{~T}$. There has been recent interest, however, in systems operating in magnetic fields of the order of $10^{-4}$ $\mathrm{T}$ (for example ${ }^{13-18}$ ), where $T_{1}$-weighted contrast is significantly enhanced ${ }^{16}\left(T_{1}\right.$ is the longitudinal relaxation time). The loss of polarization is compensated-at least in part-by 
prepolarizing ${ }^{19}$ the spins at a much higher field, or by hyperpolarization techniques using $\operatorname{lasers}^{20}$, dynamic nuclear polarization ${ }^{21,22}$ or parahydrogen-induced polarization ${ }^{23}$. The loss of signal amplitude inherent in Faraday-Law detection is mitigated by detecting the nuclear magnetization with either a Superconducting QUantum Interference Device (SQUID) ${ }^{9}$ or an atomic magnetometer ${ }^{24}$, both of which respond to the magnetic flux itself, rather than its time rate of change. Regardless of the magnitude of $B_{0}$, all currently used imaging processes involve the superposition of magnetic field gradients on a static field to impose spatial variations of the total field across the subject or sample. In the zero static field regime reported here, conventional MRI gradients are unable to encode the spins along a given direction and Fourier encoding breaks down.

In conventional MRI techniques, the applied magnetic field gradients are assumed to be linear and unidirectional so that the field due to gradients is given by $\boldsymbol{B}(x, y, z)=$ $\left(G_{\mathrm{x}} x+G_{\mathrm{y}} y+G_{\mathrm{z}} z\right) \hat{\mathbf{z}}$, where $G_{\mathrm{x}}=\partial B_{\mathrm{z}} / \partial x, G_{\mathrm{y}}=\partial B_{\mathrm{z}} / \partial y$, and $G_{\mathrm{z}}=\partial B_{\mathrm{z}} / \partial z$ are constants ${ }^{4}$. As an example, $\boldsymbol{B}(x, y, z)=G_{z} z \hat{\mathbf{z}}$ is shown in Fig. 1a. In reality, however, such idealized gradients are forbidden by the Maxwell equations $\operatorname{div} \boldsymbol{B}=\operatorname{curl} \boldsymbol{B}=0$ for any magnetic field $\boldsymbol{B}$ in free space. In fact, any gradient must be accompanied by concomitant gradients in at least one additional direction, as illustrated in Fig. 1b. At very low static fields the undesired gradient components perpendicular to $\boldsymbol{B}_{\mathbf{0}}$ induce severe, essentially intractable image distortions ${ }^{5-7}$. The degree of distortion is characterized by a parameter $\mathcal{E}=G L / B_{0}$, where $G$ is the magnitude of the field gradient and $L$ is the image field of view $(\mathrm{FOV})^{7}$. When $\varepsilon<<1$, the gradient fields can be approximated as unidirectional, greatly simplifying image encoding and reconstruction and leading to negligible image 
distortion. This "truncation" of the concomitant fields forms the basis of all MRI techniques used today including projection reconstruction and Fourier imaging ${ }^{4}$.

Several approaches have been proposed for imaging in the regime $\mathcal{E}>>1$ where conventional techniques fail ${ }^{8,25,26}$. Our experiment ${ }^{8}$ relies on the fact that, for very small angles, the precession of spins about an arbitrary field $\boldsymbol{B}$ can be represented by the sum of the precessions about each component of $\boldsymbol{B}$. After such a precession, the magnetization components that have evolved in the concomitant field can be reversed while leaving the desired unidirectional encoded component unchanged, an example of an average Hamiltonian $^{27}$.

Figure 2a shows the pulse sequence for two-dimensional imaging in the limit of zero static field, and Figs. $2 \mathrm{~b}$ and $2 \mathrm{c}$ depict the classical evolution of spins at $\left(y^{\prime}, z^{\prime}\right)$ subjected to this sequence. The proton spins are first polarized along the $x$-axis by a large field $B_{\mathrm{p}}$ which is turned off nonadiabatically ${ }^{22}$ at time $t=0$ (point $A$ in Figs. 2a, b and c). The gradient field $\boldsymbol{B}(y, z)=\left(\partial B_{\mathrm{y}} / \partial y\right) y \hat{\boldsymbol{y}}+\left(\partial B_{\mathrm{z}} / \partial z\right) z \hat{\mathbf{z}}$ is turned on, and subsequently turned off nonadiabatically at time $\tau$ (point $B$ ). During this time interval, the spin precesses about $\boldsymbol{B}\left(y^{\prime}, z^{\prime}\right)$. The time $\tau$ is chosen to satisfy the requirement $\tau<<1 / \mathcal{\gamma} G_{\mathrm{z}} L$. Consequently, the precession during the interval $\tau$ is small, and we can treat it as the sum of precessions around $\hat{\boldsymbol{z}}$ and $\hat{\boldsymbol{y}}: \delta_{\mathrm{z}}=\chi\left(\partial B_{\mathrm{z}} / \partial z\right) z^{\prime} \tau$ around $\hat{\boldsymbol{z}}$ (Fig. 2b) and $\delta_{\mathrm{y}}=$ $\chi\left(\partial B_{\mathrm{y}} / \partial y\right) y^{\prime} \tau$ around $\hat{\boldsymbol{y}}$ (Fig. 2c). After the gradient pulse, a $\pi$ pulse of uniform field $B_{\pi}$ is applied along the $z$-axis with amplitude and duration adjusted to produce a precession angle of $\pi$ around $\hat{\mathbf{z}}$. This pulse flips the spin to the point $C$ in Figs. $2 \mathrm{~b}$ and $2 \mathrm{c}$. 
Subsequently, a second gradient pulse brings the spin to $D$, and a second $\pi$ pulse to $E$. This sequence of pulses produces a net precession of the spin about $B_{\mathrm{z}}$, but no net precession about $\mathrm{B}_{\mathrm{y}}$. Thus, the two $\pi$ pulses average out the components of field perpendicular to $\hat{\mathbf{z}}$, leaving an effectively unidirectional gradient field $\boldsymbol{B}_{\text {eff }}(y, z)=G_{z} z \hat{\mathbf{z}}$.

To implement this sequence, it is convenient to define a "pulse unit" consisting of two gradient pulses and two $\pi$ pulses. Clearly, the addition of subsequent pulse units increases the angle of precession about $\hat{\mathbf{z}}$. After $n$ pulse units, the gradient has been applied for a total time $t_{\mathrm{n}}=2 n \tau$. Data are acquired at discrete values of $k$, namely $k\left(t_{\mathrm{n}}\right)=\gamma \int_{0}^{t_{\mathrm{n}}} G_{\mathrm{z}}(t) d t$, using point-by-point detection in which each point in $k$-space is acquired in a separate experiment. After the final pulse unit, a small measurement field $B_{\mathrm{m}}$ is turned on along the $z$-axis and the NMR signal from precession about this field is detected (Fig. 2d). The Fourier transform of this real-valued signal produces a complexvalued peak in frequency space, yielding the real and imaginary parts of $k(2 n \tau)$. After completing the acquisition, the $k$-space projection is Fourier transformed to obtain a onedimensional, real-space projection of the sample. Subsequently, we rotate the sample through an angle $\theta(<<\pi)$ and acquire another projection; the procedure is repeated until the range from $0^{\circ}$ to $180^{\circ}$ is covered. The image is reconstructed using filtered backprojection ${ }^{4}$.

The configuration of our experiment is shown in Fig. 3 and our results in Fig. 4. Figure 4a shows the geometry of the phantom in an image acquired in a 9.4-T MRI system with a FOV of $23 \mathrm{~mm}$. Figure $4 \mathrm{~b}$ shows the image obtained with a gradient echo 
sequence in an applied static field of $0.12 \mu \mathrm{T}$, corresponding to an NMR frequency of $\sim 5$ $\mathrm{Hz}$, applied along the $z$-axis. The image was acquired using point-by-point detection (see Supplementary Information). We estimate $\varepsilon>6.5$ (residual fields add to the applied static field). As expected, in this regime of strong concomitant gradients, Fourier encoding breaks down and the image bears no resemblance to the phantom ${ }^{5-7}$.

Figure $4 \mathrm{c}$ shows the image acquired in zero applied field with the sequence shown in Fig. 2d. We minimized the residual field $B_{\mathrm{r}}$ by performing separate NMR experiments while varying the cancellation field along the $x$-axis. The minimum NMR frequency was about $8 \mathrm{~Hz}$, corresponding to $B_{\mathrm{r}} \approx 0.2 \mu \mathrm{T}$. With a FOV $L=23 \mathrm{~mm}$, gradient pulse magnitude $G_{\mathrm{z}}=100 \mu \mathrm{T} / \mathrm{m}$, and regarding the residual field as $B_{0}$, we find $\mathcal{\varepsilon}>10$, a regime which is clearly beyond the realm of conventional MRI. Our image, however, closely resembles the high-field image. Acquisition of this image required 5.6 hours; in the Supplementary Information we illustrate how this time could potentially be reduced to a few minutes.

We can generalize our zero-field technique to the case of a uniform ambient field $B_{\mathrm{a}}$, which imposes conditions on both the gradient and $\pi$ pulses. For a given amplitude, the maximum gradient pulse duration is limited by the need to keep the precession angle small. In practice, though, we find that the zero-field sequence is quite robust-in the image shown in Fig. 4c, the maximum precession angle is approximately $65^{\circ}$. For a total field of $50 \mu \mathrm{T}$ (approximately the Earth's field), an upper bound of $65^{\circ}$ limits the maximum duration of the gradient pulse to about $85 \mu$ s (see Supplementary Information). The presence of $B_{\mathrm{a}}$ also affects the amplitude and duration of the $\pi$ pulse. Components of 
$B_{\mathrm{a}}$ perpendicular to the $\pi$ pulse induce errors by modifying the pulse amplitude and direction. To limit the error in the $\pi$ pulse to less than $1 \%$, the pulse amplitude must be approximately seven times the perpendicular component of $B_{\mathrm{a}}$. This requirement can be mitigated, however, by aligning the $\pi$ pulse with $B_{\mathrm{a}}$. Components of $B_{\mathrm{a}}$ parallel to the direction of the $\pi$ pulse, $B_{\mathrm{a}}$ (parallel), can be beneficial; the total field during the $\pi$ pulse is $B_{\pi}=B_{\pi}($ app $)+B_{\mathrm{a}}($ parallel $)$ where $B_{\pi}(a p p)$ is the applied $\pi$ pulse. Thus, if one aligns the system so that $B_{\pi}$ is parallel to (say) the Earth's field to within about $8^{\circ}$, one can keep errors in the $\pi$ pulse amplitude to less than $1 \%$ and acquire undistorted images (see Supplementary Information).

In addition to MRI, we envisage applications of our pulsed technique or related continuous-wave versions ${ }^{26}$ to experiments that use magnetic field gradients for controlling the dynamics of spins. For example, several proposals for quantum information processing ${ }^{10-12}$ use magnetic fields and field gradients to confine ions or electrons in one- ${ }^{10}$ or two-dimensional ${ }^{12}$ arrays of traps. The analysis presented by Ciaramicoli et al. ${ }^{12}$ clearly shows that the presence of concomitant gradients makes it nontrivial to address individual qubits in higher dimensional arrays. Our technique could be used ${ }^{28}$ to provide unidirectional and linear gradients in the field to address individual spins or groups of spins in three-dimensional space in a relatively straightforward manner. This addressing scheme would also enable the creation of controlled quantum Ising spin models for fundamental studies of long-range ferromagnetic interactions ${ }^{12}$ in arbitrary, user-designed lattices. 


\section{Methods}

The experimental configuration is shown schematically in Fig. 3. A doublewalled Pyrex vacuum vessel is immersed in liquid helium contained in a dewar surrounded with a single-layer mu-metal shield to attenuate external magnetic fields. A superconducting lead shield inside the dewar stabilizes the residual magnetic field. The sample rests at the bottom of the insert-which has room-temperature access-and is maintained in the liquid state at approximately $-50^{\circ} \mathrm{C}$ by a heater; the heater is switched off during encoding and data acquisition ${ }^{29}$. The coils required to generate the magnetic fields are wound from insulated $\mathrm{NbTi}$ wire and are attached to the outside of the insert. A Helmholtz pair provides a uniform field along the $z$-axis for the $\pi$ pulses, and a second Helmholtz pair, wound on top of the first, generates the measurement field $B_{\mathrm{m}}$. The rectangular gradient coils generate a field of the form $\boldsymbol{B}(y, z)=\left(\partial B_{\mathrm{y}} / \partial y\right) y \hat{\boldsymbol{y}}+\left(\partial B_{\mathrm{z}} / \partial z\right) z \hat{\mathbf{z}}$ in the $y-z$ plane (shown in Fig. 1b) over the imaging FOV, where $\partial B_{\mathrm{y}} / \partial y \approx-0.9\left(\partial B_{\mathrm{z}} / \partial z\right)$; since we image in the $y-z$ plane, we neglect the effects of gradients along $\hat{\boldsymbol{x}}$. A further pair of coils largely cancels the residual field from the Earth, which is predominantly along the $x$-axis. The signal from the precessing spins is detected by a first-derivative, superconducting gradiometer coupled to the input coil of a Nb-based SQUID ${ }^{9}$. The gradiometer, which consists of two $\mathrm{Nb}$-wire loops of nominally equal area wound in opposite senses and connected in series, reduces ambient noise in the measurement direction. A series array of 24 Josephson junctions limits the supercurrent while the fields are being switched ${ }^{30}$. The SQUID is enclosed in a Nb shield suspended below the insert, and is read out using a flux-locked loop 9 


\section{References}

1. Lauterbur, P. Image formation by induced local interactions: Examples employing nuclear magnetic resonance. Nature 242, 190-191 (1973).

2. Mansfield, P. \& Morris, P. G. NMR Imaging in Biomedicine (Academic Press, London, 1982).

3. Callaghan, P. T. Principles of Nuclear Magnetic Resonance Microscopy (Oxford Univ. Press, New York, 1993).

4. Haacke, E. M., Brown, R. W., Thompson, M. R. \& Venkatesan, R. Magnetic Resonance Imaging: Physical Principles and Sequence Design (Wiley, New York, 1999).

5. Norris, D. G. \& Hutchinson, J. M. S. Concomitant magnetic field gradients and their effects on imaging at low magnetic field strengths. Magn. Reson. Imaging 8, 33-37 (1990).

6. Bernstein, M. A. et al. Concomitant gradient terms in phase contrast MR: Analysis and correction. Magn. Reson. Med. 39, 300-308 (1998).

7. Yablonskiy, D. A., Sukstanskii, A. L. \& Ackerman, J. J. H. Image artifacts in very low magnetic field MRI: The role of concomitant gradients. J. Magn. Reson. 174, 279-286 (2005).

8. Meriles, C. A., Sakellariou, D., Trabesinger, A. H., Demas, V. \& Pines, A. Zeroto low-field MRI with averaging of concomitant gradient fields. Proc. Natl. Acad. Sci. U.S.A. 102, 1840-1842 (2005). 
9. Clarke, J. \& Braginski, A. I. (eds.) The SQUID Handbook Vol. I: Fundamentals and Technology of SQUIDs and SQUID Systems (Wiley-VCH, Weinheim, 2004).

10. Mc Hugh, D. \& Twamley, J. Quantum computer using a trapped-ion spin molecule and microwave radiation. Phys. Rev. A 71, 012315 (2005).

11. Wunderlich, C., Morigi, G. \& Reiss, D. Simultaneous cooling of axial vibrational modes in a linear ion trap. Phys. Rev. A 72, 023421 (2005).

12. Ciaramicoli, H., Marzoli, I. \& Tombesi, P. Quantum spin models with electrons in Penning traps. Phys. Rev. A 78, 012338 (2008).

13. Callaghan, P. T. \& Le Gros, M. Nuclear spins in the Earth's magnetic field. Am. J. Phys. 50, 709-713 (1982).

14. Stepišnik, J., Eržen, V. \& Kos, M. NMR imaging in the earth's magnetic field. Magn. Reson. Med. 15, 386-391 (1990).

15. Xu, S. et al. Magnetic resonance imaging with an optical atomic magnetometer. Proc. Natl. Acad. Sci. U.S.A. 103, 12668-12671 (2006).

16. Clarke, J., Hatridge, M. \& Mößle, M. SQUID-detected magnetic resonance imaging in microtesla fields. Annu. Rev. Biomed. Eng. 9, 389-413 (2007).

17. Zotev, V. S. et al. Microtesla MRI of the human brain combined with MEG. $J$. Magn. Reson. 194, 115-120 (2008).

18. Blümich, B. The incredible shrinking scanner. Sci. Am. 299, $92-96$ (November 2008).

19. Packard, M. \& Varian, R. Free nuclear induction in the earth's magnetic field. Phys. Rev. 93, 941 (1954). 
20. Happer, W. et al. Polarization of the nuclear spins of noble-gas atoms by spin exchange with optically pumped alkali-metal atoms. Phys. Rev. A 29, 3092-3110 (1984).

21. Overhauser, A. W. Polarization of nuclei in metals. Phys Rev. 92, 411-415 (1953).

22. Abragam, A. Principles of Nuclear Magnetism (Oxford Univ. Press, New York, 1989).

23. Bowers, C. R. \& Weitekamp, D. P. Transformation of symmetrization order to nuclear-spin magnetization by chemical reaction and nuclear magnetic resonance. Phys. Rev. Lett. 57, 2645-2648 (1986).

24. Budker, D., \& Romalis, M. Optical magnetometry. Nature Physics 3, 227-234 (2007).

25. Meriles, C. A., Sakellariou, D., Trabesinger, A. H. Theory of MRI in the presence of zero to low magnetic fields and tensor imaging field gradients. J. Magn. Reson. 182, 106-114 (2006).

26. Bouchard, L.-S. Unidirectional magnetic-field gradients and geometric-phase errors during Fourier encoding using orthogonal ac fields. Phys. Rev. B 74, 054103 (2006).

27. Haeberlen, U. \& Waugh, J. S. Coherent averaging effects in magnetic resonance. Phys. Rev. 175, 453-467 (1968).

28. Slice and volume-selective rotations can be performed in a manner similar to the method proposed in Ref. (26). 
29. The sample was maintained at $-50^{\circ} \mathrm{C}$ to avoid dissipating too much power in the heater; there is no inherent difficulty with imaging a room temperature sample with a cryogenic detector ${ }^{16}$.

30. Hilbert, C., Clarke, J., Sleator, T. \& Hahn, E. L. Nuclear quadrupole resonance detected at $30 \mathrm{MHz}$ with a dc superconducting quantum interference device. Appl. Phys. Lett. 47, 637-639 (1985).

\section{Acknowledgments}

This work was supported by the Director, Office of Science, Office of Basic Energy

Sciences, Materials Science and Engineering Division, of the U.S. Department of Energy under Contract No. DE-AC02-05CH1 1231. The authors thank S. Conolly, J. Reimer and D. Wemmer for thoughtful comments on the manuscript, and S. Busch, M. Hatridge and M. Moessle for helpful discussions. 


\section{Figure Legends}

Fig. 1 Idealized and achievable magnetic field gradients. a, Idealized gradient field $\boldsymbol{B}=$ $\left(\partial B_{\mathrm{z}} / \partial z\right) z \hat{\mathbf{z}}$. Such a field violates Maxwell's equations. b, Example of a realizable gradient field in the $y-z$ plane of the form $\boldsymbol{B}(y, z)=\left(\partial B_{\mathrm{y}} / \partial y\right) y \hat{\boldsymbol{y}}+\left(\partial B_{\mathrm{z}} / \partial z\right) z \hat{\boldsymbol{z}}$. Lengths of vectors represent relative field strengths.

Fig. 2 Protocol for MRI in zero static field. a, Pulse sequence vs. time. b-c, Progression of the spin vector at times $t=0(A), \tau(B), 2 \tau(C), 3 \tau(D)$ and $4 \tau(E)$ about (b) $z$-axis and (c) $y$-axis. d, Pulse sequence used for the zero-field MRI experiment differs from that in b in two respects. First, after the final pulse pair, a gradient pulse was applied for a time $\tau / 2$; this pulse corrects higher order errors ${ }^{8,25}$. Second, to ensure that the important $k=0$ point was included, the gradient was inverted in the first pulse unit, so that the first point in $k$-space was $k(5 \tau / 2)=-(3 \tau / 2) \gamma G_{\mathrm{z}}$. All subsequent gradient pulses have positive polarity; for example, the second $k$-space point was $k(9 \tau / 2)=(\tau / 2) \gamma G_{\mathrm{z}}$. Note that the measurement field $B_{\mathrm{m}}$ is not applied during encoding pulses; it is used solely for pointby-point $k$-space acquisition, enabling quadrature detection with a single sensor.

Fig. 3 Configuration of experiment.

Fig. 4 Images of a phantom. Views are along the axis of a nylon cylinder $17 \mathrm{~mm}$ in diameter and $35 \mathrm{~mm}$ long in which a cavity has been cut and filled with water (a) or ethanol (b,c). a, High-field conventional image acquired at 9.4 T. b, Conventional 
gradient-echo image with $\varepsilon>6.5$ bears no relation to the phantom due to concomitant field distortions. c, Image encoded in the approach to zero applied static field where the concomitant fields of the encoding gradients yield $\mathcal{E}>10$. The prepolarization field $B_{\mathrm{p}} \approx$ $10 \mathrm{mT}$ was applied for $2 \mathrm{~s}$. The image was encoded in nearly zero static field using 100$\mu \mathrm{T} / \mathrm{m}$ gradient pulses with a duration $\tau=5 \mathrm{~ms}$. The $\pi$ pulses, with a magnitude of approximately $12 \mu \mathrm{T}$ and duration of $1 \mathrm{~ms}$, produced an effective field $\boldsymbol{B}_{\text {eff }}=\left(\partial B_{z} / \partial z\right) z \hat{\mathbf{z}}$. The $\pi$ pulse amplitude was determined in separate experiments to an accuracy of $\pm 1 \%$. After the spins were encoded, the NMR signal was acquired in $1 \mathrm{~s}$ in a measurement field $B_{\mathrm{m}}=3.75 \mu \mathrm{T}$, corresponding to an NMR frequency of $160 \mathrm{~Hz}$. Projections were acquired every $7.5^{\circ}$, so that 24 projections covered the range from $0^{\circ}$ to $172.5^{\circ}$. The time for each projection was about $14 \mathrm{~min}$, giving a total acquisition time of about $5.6 \mathrm{~h}$. Each $k$-space projection contained 24 points. 

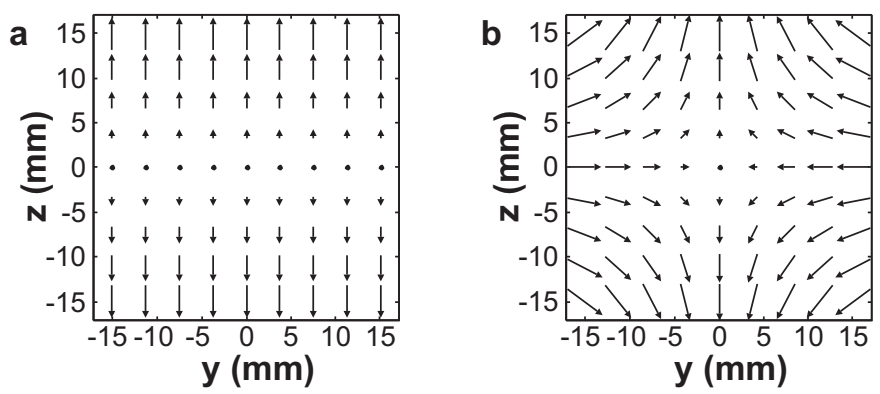

Figure 1 


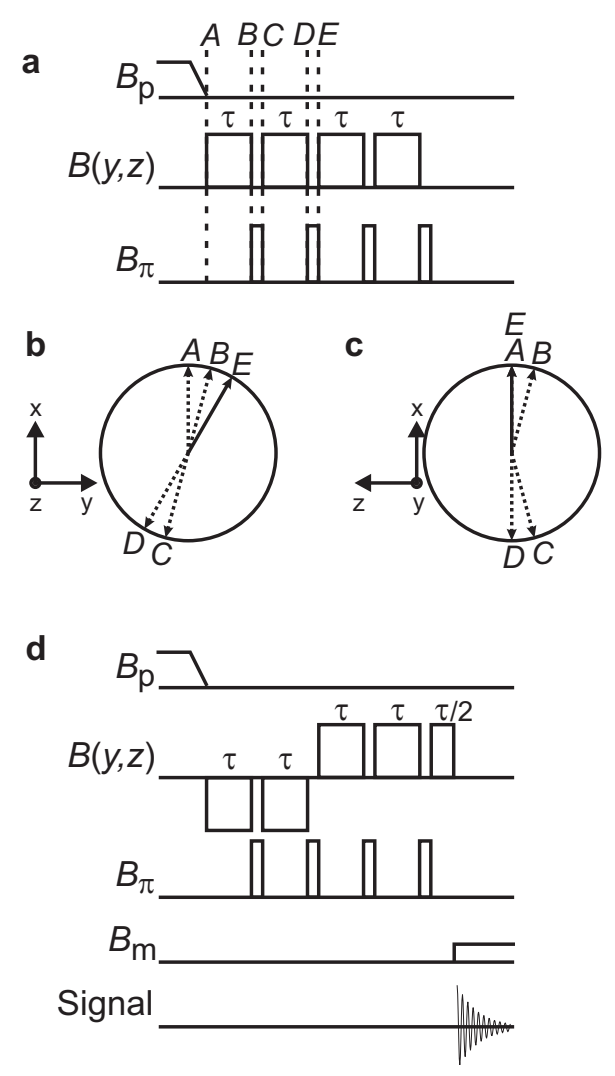

Figure 2 


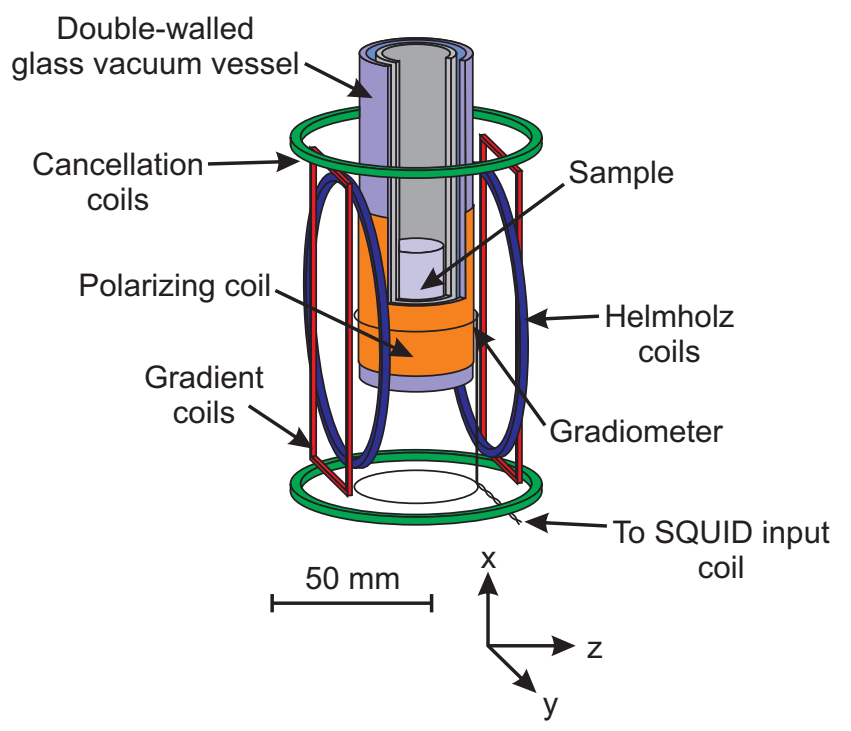

Figure 3 

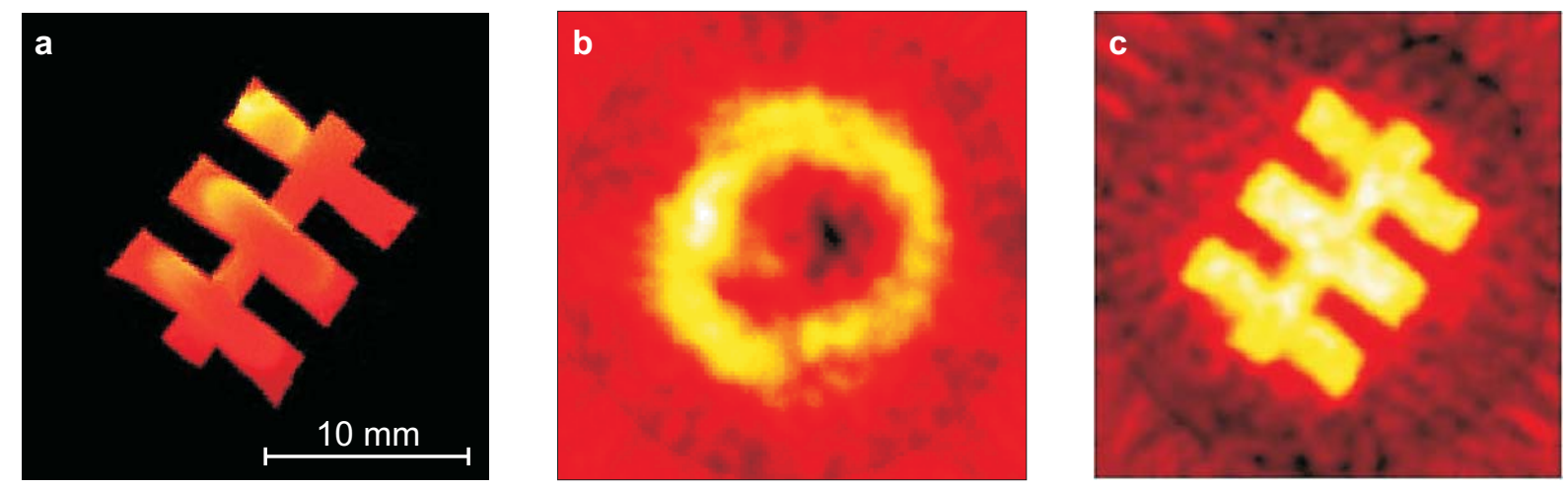

Figure 4 


\section{Supplementary Information}

\section{Gradient Echo Imaging}

The image shown in Fig. 4c was acquired using a conventional gradient echo sequence ${ }^{4}$ modified for point-by-point $k$-space acquisition. The sequence used to acquire the $k(9 \tau / 2)$ point is shown in Fig. S1. The proton spins are first polarized along the $x$-axis by a large field $B_{\mathrm{p}}$ which is turned off nonadiabatically at time $t=0$. Subsequently, two fields are switched on: a uniform static field $B_{0}$ along the $z$-axis, and a negative gradient field $-\boldsymbol{B}(y, z)=-\left(\partial B_{\mathrm{y}} / \partial y\right) y \hat{\boldsymbol{y}}-\left(\partial B_{z} / \partial z\right) z \hat{\mathbf{z}}$. After a time $2 \tau$ the gradient is reversed. The $B_{0}$ and gradient fields are maintained until the desired point in $k$-space is reached, at which time a measurement field $B_{\mathrm{m}}$ is applied. The time-domain data are acquired and processed as described in the main text for the zero-field experiment.

The point-by-point $k$-space acquisition technique was used in the conventional image in order to match as closely as possible the conditions for the zero-field image. The conventional imaging experiment uses the same polarizing pulse, gradients, and encoding times as does the zero-field experiment. The gradient echo sequence requires a uniform static field $B_{0}$ during encoding in order to establish a "preferred" gradient direction; components of the gradient perpendicular to $B_{0}$ are the unwanted concomitant terms. (Recall that in the zero-field sequence, the preferred gradient direction is that of the $\pi$ pulses.) 


\section{Imaging Time Considerations}

Our implementation of zero-field MRI involves acquiring $k$-space point-by-point. The primary reason is that this method yields the real and imaginary parts of $k$-space, effectively providing quadrature detection with a single sensor, but the procedure is time

consuming. For each of the 24 projections of the image we acquire 24 points, each taking 3.5 seconds (2-s polarizing pulse followed by up to 0.5 -s encoding time and 1 -s data acquisition time), and each point is averaged 10 times to increase the signal-to-noise ratio (SNR) leading to a total acquisition time of about 5.6 hours. The imaging time could be reduced substantially by using two orthogonal SQUID-based gradiometers. Since all points in one projection could be acquired in one experiment, the imaging time would be reduced by a factor of 24 . Increasing the prepolarization field from $10 \mathrm{mT}$ to $100 \mathrm{mT}$ would increase the SNR 10-fold. As shown below, these two factors alone would reduce the acquisition time to 2-3 minutes. Adding a Helmholtz pair along the $y$-axis (to allow $\pi$ pulses in an arbitrary direction in the $y$-z plane) and a second, off-diagonal gradient such as $\partial B_{\mathrm{z}} / \partial y$ would make it possible to perform acquisitions along arbitrary $k$-space trajectories, and would eliminate the need to rotate the sample. Optimized $k$-space sampling would result in further improvements in image quality and acquisition time.

We now outline our calculations of the statements above. In MRI, the SNR is commonly defined as the signal amplitude divided by the standard deviation of the noise. For an acquisition lasting a time of $t_{a c q}$ 


$$
S N R=\frac{\int_{0}^{t_{a c q}} s(t) d t}{\sqrt{\int_{0}^{t_{a c q}} \sigma_{n}^{2} d t}}=\left[\frac{\int_{0}^{t_{\text {acq }}} s(t) d t}{\sqrt{t_{a c q}}}\right] \frac{1}{\sigma_{n}},
$$

where $\sigma_{n}$ is the standard deviation of the noise (which is stationary and assumed to originate from the electronics and detector) and $s(t)$ is the integrated signal from the nuclear magnetization detected by the sensor (assuming negligible noise) ${ }^{4}$. To compare the SNR of two acquisition methods, we estimate the value of the bracketed term from the formula

$$
s(t) \propto \iint_{y} m(y, z) \exp \left[-t / T_{2}^{*}\right] \exp \left[-i \not t B_{G}(y, z)\right] d y d z,
$$

where $T_{2} *$ is the transverse relaxation time, $B_{\mathrm{G}}(y, z)$ is the field due to applied gradients, and $m(y, z)$ is a function representing the spin distribution in the sample, normalized such that

$$
\iint_{y} m(y, z) d y d z=1
$$

In the point-by-point (pbp) acquisition method described in the text, the signal is acquired as a free induction decay (FID) in the uniform field $B_{\mathrm{m}}$. The demodulated signal equation in this case is given by

$$
S_{p b p}(t)=\iint_{y} m(y, z) \exp \left[-t / T_{2}^{*}\right] d y d z,
$$

where, in our experiments, the transverse relaxation time $T_{2}{ }^{*}$ was measured to be $300 \mathrm{~ms}$. Using our acquisition time $t_{\mathrm{acq}}=1$ second, 


$$
S N R_{p b p} \propto \frac{\int_{0}^{t_{a c q}} s_{p b p}(t) d t}{\sqrt{t_{a c q}}} \approx 0.289 .
$$

In a directly-detected experiment using two orthogonal detectors, the signal could be detected as precession about the gradient field $\boldsymbol{B}_{\mathbf{G}}$ during every second gradient pulse. The demodulated signal equation in this case is given by

$$
s_{d i r}(t)=\iint_{y} m(y, z) \exp \left[-t / T_{2}^{*}\right] \exp \left[-i \not t B_{G}\right] d y d z,
$$

where

$$
B_{G}=\sqrt{\left[\left(\partial B_{y} / \partial y\right) y\right]^{2}+\left[\left(\partial B_{z} / \partial z\right) z\right]^{2}}
$$

is the field magnitude at the point $(y, z)$. For our value $B_{\mathrm{G}}=100 \mu \mathrm{T} / \mathrm{m}$ and a 5 -ms acquisition

$$
S N R_{d i r} \propto \frac{\sqrt{2} \int_{0}^{t_{a c q}} s_{d i r}(t) d t}{\sqrt{t_{a c q}}} \approx 0.0990 .
$$

The factor of $\sqrt{2}$ arises from the use of two detectors in quadrature detection.

We now compare the difference in SNR between the two acquisition methods. If the noise standard devation $\sigma_{n}$ is the same in both cases, we find

$$
\frac{S N R_{d i r}}{S N R_{p b p}} \propto \frac{0.0990}{0.289} \approx 0.34 .
$$

The SNR in the directly-detected experiment is about $1 / 3$ that of the point-by-point experiment, while the imaging time is reduced by a factor of 24 . 
As stated in the main text, our point-by-point imaging procedure took approximately 5.6 hours; the time is long because each $k$-space point is acquired in a separate experiment. The imaging time could potentially be reduced to approximately two minutes, for the equivalent SNR, by acquiring the signal directly (using two orthogonal detectors), eliminating signal averaging, and increasing the polarizing field from $10 \mathrm{mT}$ to $100 \mathrm{mT}$.

We explain this estimate as follows. The direct acquisition method would reduce imaging time by a factor of 24 by acquiring all $k$-space points in a single experiment. The SNR loss, however, is a factor of $\sim 3$, as described previously. Elimination of signal averaging reduces imaging time by an additional factor of 10 , at the cost of an additional factor of $\sim 3$ in SNR. Thus, the total SNR drops by a factor of $\sim 9$ while reducing imaging time by a factor of 240 . This factor of $\sim 9$ loss in SNR can be recovered by increasing the prepolarization field from $10 \mathrm{mT}$ to $100 \mathrm{mT}$. Together, these factors result in the same SNR, but with a substantial reduction in acquisition time.

\section{Effect of a Uniform Ambient Field on Gradient Pulse Duration}

The angle of precession during a field pulse of amplitude $B$ and duration $\tau$ is given (in radians) by $\delta=\gamma B \tau$. Solving for $\tau$ with $\delta=65^{\circ} \approx 1.13$ radians, $\gamma=2 \pi * 42.6 \mathrm{~Hz} / \mu \mathrm{T}$, and $B=50 \mu$ T yields $\tau \approx 85 \mu \mathrm{s}$.

Effect of a Uniform Ambient Field on $\pi$ Pulse Amplitude 
We consider the conditions on the $\pi$ pulse imposed by the presence of a uniform ambient field. The $z$-axis is defined to be along the direction of the applied $\pi$ pulse. The $z$-axis is thus the desired direction of the total $\pi$ pulse; in the presence of an ambient field, the field amplitude along $z$ is given by $B_{\pi}(a p p)+B_{\mathrm{a}}$ (parallel) where $B_{\pi}(a p p)$ is the applied pulse and $B_{\mathrm{a}}$ (parallel) is the component of ambient field parallel to the z-axis.

For a component of ambient field perpendicular to the $z$-axis $B_{\mathrm{a}}($ perp $)$, the total field during the $\pi$ pulse is given by (Fig. S2)

$$
B_{\pi}(\text { tot })=\sqrt{\left[B_{\pi}(\text { app })+B_{a}(\text { parallel })\right]^{2}+\left[B_{a}(\text { perp })\right]^{2}} .
$$

Thus $B_{\pi}($ tot $) \leq 1.01\left[B_{\pi}(\right.$ app $)+B_{\mathrm{a}}($ parallel $\left.)\right]$ when $B_{\mathrm{a}}($ perp $) \leq B_{\pi}($ tot $) / 7$.

For the limiting case in which $B_{\pi}(a p p)=0$, the angle $\theta$ in Fig. S2 gives the misalignment between $B_{\mathrm{a}}$ and the $z$-axis. To maintain the condition $B_{\mathrm{a}}($ perp $) \leq B_{\pi}(t o t) / 7$, one requires $\theta \leq 8^{\circ}$. 


\section{Figure Captions for Supplementary Information}

Fig. S1. Pulse sequence vs. time for the $k(9 \tau / 2)$ point of the conventional image.

Fig. S2. Effect of uniform ambient field on $\pi$ pulse. 


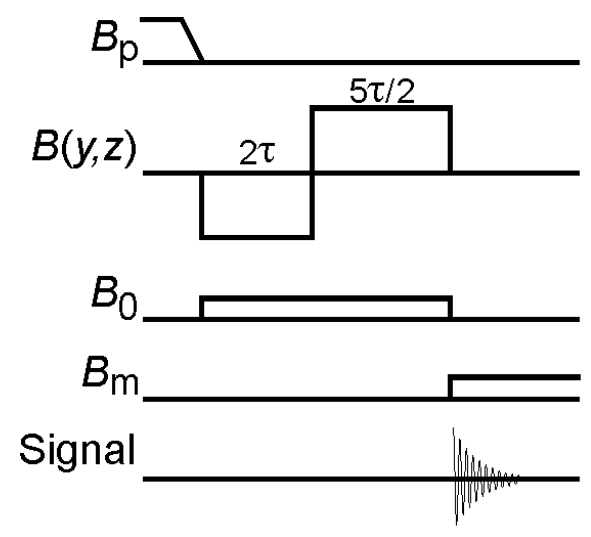

Fig. S1 


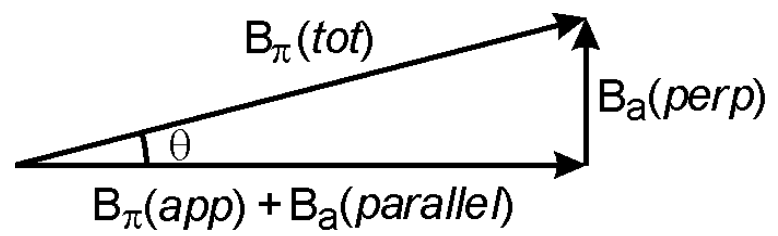

Fig. S2 


\section{Disclaimer}

This document was prepared as an account of work sponsored by the United States Government. While this document is believed to contain correct information, neither the United States Government nor any agency thereof, nor The Regents of the University of California, nor any of their employees, makes any warranty, express or implied, or assumes any legal responsibility for the accuracy, completeness, or usefulness of any information, apparatus, product, or process disclosed, or represents that its use would not infringe privately owned rights. Reference herein to any specific commercial product, process, or service by its trade name, trademark, manufacturer, or otherwise, does not necessarily constitute or imply its endorsement, recommendation, or favoring by the United States Government or any agency thereof, or The Regents of the University of California. The views and opinions of authors expressed herein do not necessarily state or reflect those of the United States Government or any agency thereof or The Regents of the University of California. 